\title{
Biosynthesis of Ethylene
}

\section{DUAL NATURE OF COFACTOR REQUIRED FOR THE ENZYMIC PRODUCTION OF ETHYLENE FROM METHIONAL}

\author{
By L. W. MAPSON AND A. MEAD \\ Agricultural Research Council, Food Research Institute, Low Temperature Research Station, Cambridge
}

(Received 19 February 1968)

\begin{abstract}
The heat-stable cofactor in cauliflower florets, which has been shown to be necessary for the enzymic production of ethylene from methional, consists of two components. The first is of a phenolic nature and appears to be an ester of $p$ coumaric acid. The second component is acidic in character, but has not as yet been identified.
\end{abstract}

A heat-stable cofactor is necessary for the conversion of methional (3-methylthiopropionaldehyde) into ethylene by the methional-cleaving enzyme from cauliflower florets (Mapson \& Wardale, 1967). This cofactor occurs in deproteinized aqueous extracts from floret tissue. Fractionation of these extracts has now been carried out in an attempt to isolate and characterize the nature of the substance or substances involved.

\section{EXPERIMENTAL}

Reagents. Polyamide (Woelm TLC) was purchased from M. Woelm, Eschwege, Germany, and was used to separate the acidic component (fraction $A$ ) from the phenolic component (fraction $C$ ). The polyamide was made into a column $(12 \mathrm{~cm} . \times 2.5 \mathrm{~cm}$.) and washed with distilled water. The ether-soluble organic acids were applied to the top and fractions successively eluted with water, $0 \cdot 1 \mathrm{~m}-\mathrm{NaHCO}_{3}$ and $2 \mathrm{~N}-\mathrm{NH}_{3}$. The column was regenerated for further use by washing with $2 \mathrm{~N}-\mathrm{NaOH}$ to remove the last traces of phenols and finally with water. Hexamethyldisilazane and trimethylchlorosilane were obtained from Koch-Light Laboratories Ltd., Colnbrook, Bucks. Pyridine (AnalaR grade; British Drug Houses Ltd., Poole, Dorset) was redistilled and stored over $\mathrm{KOH}$ pellets. These reagents, however, had to be purified just before use. Methanol was dried and purified by distillation from a mixture of the Grignard derivative.

Enzymic tests. The activity of the unknown cofactors were estimated by following the rate of production of ethylene from methional in the presence of glucose-glucose oxidase and the methional-cleaving enzyme (Mapson \& Wardale, 1968). To test the activity of the acidic component (fraction $A$ ) $p$-hydroxybenzoate was used to supply the phenolic component (fraction $C$ ). To test the activity of the phenolic component in fraction $C$ the acidic component isolated in fraction $A$ or in the later purified fractions was used.

Preparation of trimethylsilyl derivatives. The component in the extract not adsorbed on polyamide (fraction $A$ ) was first purified by chromatography on paper (Whatman no. 1) with propan-1-ol-aq. $\mathrm{NH}_{3}$ (sp.gr. $\left.0 \cdot 88\right)(7: 3, \mathrm{v} / \mathrm{v}$ ) as solvent. The acids were located by spraying with universal indicator (British Drug Houses Ltd.) containing $2 \mathrm{ml}$. of $0.1 \mathrm{~N}$ $\mathrm{NaOH} / 25 \mathrm{ml}$. of indicator solution. The active component $\left(R_{F}\right.$ approx. 0.5$)$ was eluted from the paper with either water or methanol, evaporated to dryness and the residue dried over $\mathrm{P}_{2} \mathrm{O}_{5}$ for 2-3 hr. under vacuum.

Silyl derivatives were prepared in stoppered test tubes by the method of Dalgliesh, Horning, Horning, Knox \& Yarger (1966). They were found to be stable in the presence of pyridine and excess of reagents. The derivatives were formed fairly rapidly and injections of a derivative into the gas chromatograph could be made $1-2 \mathrm{hr}$. after addition of reagents. Quantitative conversion did not occur under these conditions, but the amount formed was adequate for preparative gas-liquid chromatography.

Instrumental details. The instrument used was a series 104 model 24 dual flame ionization gas chromatograph (W. G. Pye and Co., Cambridge). One channel had been adapted to preparative use by the addition of a Pye manual preparative attachment.

The carrier gas (argon) was used at a flow rate of $80 \mathrm{ml}$./ min. for preparative work and at $40 \mathrm{ml}$. $/ \mathrm{min}$. for analytical work. The column for analytical work was a $5 \mathrm{ft} . \times \frac{1}{4}$ in. coiled glass column packed with $3 \%$ methyl silicone gum (SE 30) on 100-120-mesh Celite. The preparative column was a $7 \mathrm{ft} . \times \frac{3}{8}$ in. coiled glass column packed with $25 \%$ SE 30 on 60-72-mesh silicone-treated Celite.

The sample for preparative work was injected at a column temperature of $100^{\circ}$ and programmed linearly at $4^{\circ} / \mathrm{min}$. up to $250^{\circ}$, from the appearance of the solvent peak front. The flash heater at the injection point was maintained approx. $50^{\circ}$ above the oven temperature. This corresponded to a 'MAX' setting on the Pye thermoregulator. The outlet heater at the sample-collection point was also maintained at the ' $M A X$ ' setting. Though its temperature was never measured it was sufficient to allow satisfactory trapping of the components of interest.

Trapping procedures. The traps used were standard Pye $7 \mathrm{~mm}$. glass U-tube traps. For this experiment they were packed with dry 80-mesh glass beads (British Drug 


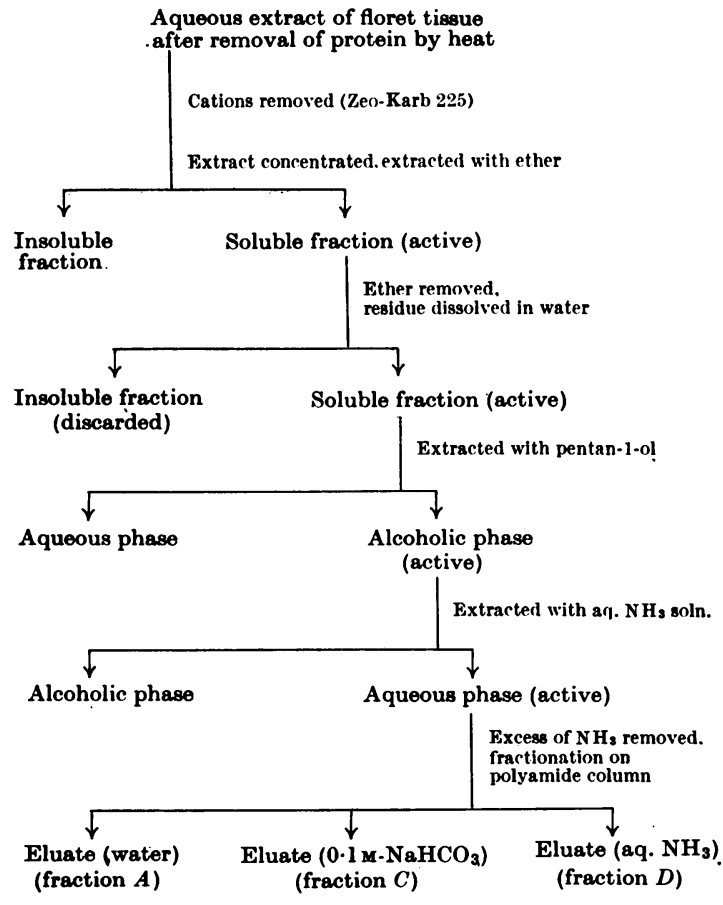

Scheme 1. Preliminary stages in the fractionation of cofactors from cauliflower floret tissue.

Houses Ltd.), cleaned before use with chromic acid, water and pure methanol, and plugged with quartz wool.

After being packed the traps were dried at $100^{\circ}$ for $2 \mathrm{hr}$. and allowed to cool in a desiccator over anhydrous $\mathrm{CaCl}_{2}$. This procedure was carried out to remove all traces of moisture from the traps, since this would otherwise have led to hydrolysis of the silyl derivatives.

During use the traps were fitted with small $\mathrm{CaCl}_{2}$ drying tubes on their outlets to prevent intake of moisture. The traps were corked at their inlets and cooled in an acetonesolid $\mathrm{CO}_{2}$ bath for $20 \mathrm{~min}$. before use. The corks were removed and the traps attached to the collection part on the chromatograph at the onset of the peak of interest. During the entire collection period the traps were kept cold by immersion in the acetone-solid $\mathrm{CO}_{2}$ bath. After collection the trap was removed from the chromatograph and the $\mathrm{CaCl}_{2}$ tube removed from the trap. The silyl derivative was eluted from the trap with methanol, the methanol removed in vacuo and the silyl derivative hydrolysed by the addition of water and warming at $60-80^{\circ}$ for $5 \mathrm{~min}$.

\section{RESULTS}

Preliminary observations indicated that an increase in the ability of aqueous extracts to promote the enzymic formation of ethylene from methional occurred if the solutions prepared from the florets by extraction with cold water were allowed to autolyse for $24-28 \mathrm{hr}$. in the presence of air or oxygen. Under such conditions the activity of the extracts increased two- to three-fold compared with an extract that was heated at $100^{\circ}$ immediately after extraction of the floret tissue. The steps in the fractionation procedure subsequent to removal of protein after heat coagulation are outlined in Scheme 1.

Cations in the extract were removed by passage through Zeo-Karb $225\left(\mathrm{H}^{+}\right.$form) and the resulting effluent was concentrated in vacuo to a small bulk. This was then admixed with Celite and the semi-dry powder extracted for $12-24 \mathrm{hr}$. in a Soxhlet apparatus with diethyl ether. The ethereal solution was evaporated to dryness and the residue dissolved in water. Insoluble material, which was inactive, was discarded. The aqueous acid extract was extracted in two successive operations with an equal volume of pentan-1-ol. The acids in the pentan-1-ol-soluble material were rendered watersoluble by subsequent extraction with ammoniacal solution ( $\mathrm{pH}$ approx. 10). Excess of ammonia was removed by concentration in vacuo and the solution of ammonium salts ( $\mathrm{pH}$ approx. 6.3) was added to a column of polyamide previously washed with water.

Separation of two cofactors on polyamide. Each fraction in the foregoing procedure stimulated the production of ethylene from methional when added in concert with the methional-cleaving enzyme, glucose oxidase and glucose. After fractionation on polyamide, however, separation of two distinct fractions was achieved. Singly these were inactive, but they induced activity when added together to the enzymic system. These two fractions were separated by chromatography on a polyamide column as follows. The column $(12 \mathrm{~cm} . \times 2.5 \mathrm{~cm}$.) was prepared by first washing with water, and after the addition of the acids as ammonium salts the chromatogram was developed first by elution with water and was continued until the slightly yellow effluent ceased (fraction $A$ ). Elution with water was continued until a further $50-60 \mathrm{ml}$. of effluent had passed (fraction $B$ ).

The column was then eluted with $0 \cdot 1 \mathrm{M}$-sodium hydrogen carbonate, $\mathrm{pH} 8 \cdot 3$, and the elution continued until the effluent was colourless (fraction $C)$. Finally the bulk of the phenolic acids were eluted with $2 \mathrm{~N}$-ammonia solution until the eluate was again colourless (fraction $D$ ).

The ability of the fractions so isolated to promote ethylene formation from methional was tested; the results are shown in Fig. 1. It is clear that none of the fractions was active singly, only fractions $A$ and $C$ when added together. Fraction $B$ alone or in combination with other fractions had no effect, and fraction $D$, containing most of the polyphenols, though inactive itself, was inhibitory when admixed 


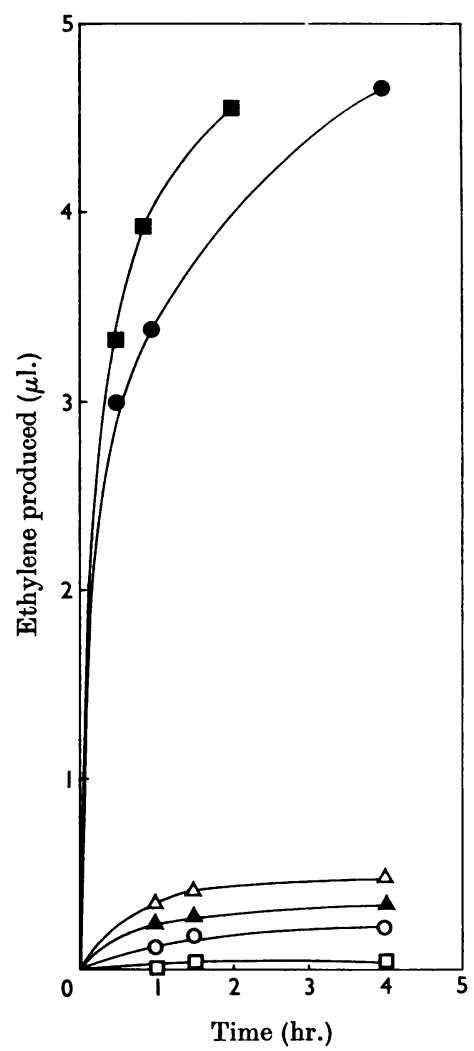

Fig. 1. Separation of the two components of the cofactor on polyamide. Enzyme system contained $8 \mathrm{ml}$. of $\mathrm{KH}_{2} \mathrm{PO}_{4}-$ $\mathrm{Na}_{2} \mathrm{HPO}_{4}$ buffer, pH6.5 (0.1 M), EDTA (2 mM), methional (1 mM), glucose (50 mg.), glucose oxidase $(4 \mu \mathrm{g}$.$) and 1 \mathrm{ml}$. of a protein fraction precipitated at $30-60 \%$ saturation with $\left(\mathrm{NH}_{4}\right)_{2} \mathrm{SO}_{4}$ to supply the methional-cleaving enzyme. The fractions $A, B, C$ or $D$ eluted from the polyamide column were added as indicated. $\square$, Untreated extract; $\triangle$, fractions $B$ and $C$;, fractions $A$ and $C ; \square$, fraction $D$; ○, fraction $A ; \triangle$, fraction $C$.

with fractions $A$ and $C$. The heat-stable cofactor in the aqueous extract thus consists of two distinct components, both of which are essential for the enzymic reaction.

Nature of the active component in fraction $\mathrm{C}$. Separation of the various components eluted by $0 \cdot 1 \mathrm{M}$-sodium hydrogen carbonate, $\mathrm{pH} \mathrm{8.3,} \mathrm{from}$ polyamide columns was attempted by chromatography on paper with aqueous $2 \%(v / v)$ acetic acid as solvent. The phenolic compounds on the chromatograms were identified either (1) by their fluorescence in ultraviolet light before and after treatment with ammonia or (2) by their reaction with diazotized $p$-nitroaniline. The areas giving positive reactions with either test were eluted with
$0.1 \mathrm{M}$-phosphate buffer, $\mathrm{pH} 6.5$, and the activity of the extract was tested with the addition of the component contained in fraction $A$ in the enzymic test. In many experiments the active component was associated with a single area of the chromatogram that was not fluorescent in u.v. light, but fluorescing light-blue after treatment with ammonia, and giving a purple colour with a $p$-nitroaniline spray. In later experiments with extracts from other cauliflowers and with the same treatment, sometimes two and sometimes three distinct fluorescent bands were observed. All of these bands when extracted and tested enzymically were active. On being rechromatographed with propan1-ol-aq. ammonia (sp.gr. 0.88) $(3: 2, \mathrm{v} / \mathrm{v})$ these bands $\left(R_{F} \quad 0.66\right)$ appeared as one band. This procedure had the advantage of freeing them from other contaminating phenols fluorescing yellow in u.v. light after treatment with ammonia. This difference in behaviour between extracts from different cauliflowers we believe to be due to variation in the composition of these compounds and not to a failure to resolve these different components in those cases in which only one fluorescent band was observed. The separate fluorescent bands were purified by chromatography on paper first with propan-1-ol-aq. ammonia (sp.gr. 0.88) $(3: 2, \mathrm{v} / \mathrm{v})$ followed by $2 \%(\mathrm{v} / \mathrm{v})$ acetic acid. The u.v. spectra of these compounds in methanol were determined. These in each case closely resembled those of esters of $p$-coumaric acid. This resemblance with authentic esters of $p$ coumaric acid (methyl, glucose or quinic esters) occurred not only in neutral solutions but in the extent of the shift of the absorption maxima on making them alkaline (Fig. 2). Confirmation that these unknown compounds were derivatives of $p$. coumaric acid was obtained in the following manner. The active components were isolated after chromatography on paper first with $2 \%(\mathrm{v} / \mathrm{v})$ acetic acid as solvent, followed by chromatography with propan-1-ol-aq. ammonia (sp.gr. 0.88) as solvent. The eluted esters were hydrolysed by treatment with $2 \mathrm{~N}$-sodium hydroxide for $2 \mathrm{hr}$. at room temperature. Phenols were extracted after acidification with ethyl acetate and again chromatographed with $2 \%(\mathrm{v} / \mathrm{v})$ acetic acid. In each case $p$-coumaric acid was identified in both its trans-and cis-forms $\left(R_{F} 0.35\right.$ and 0.68 respectively) from each of the unknown esters, by (1) their reaction in u.v. after treatment with ammonia (deep-violet fluorescence), (2) their reaction to diazotized $p$-nitroaniline (blue) and (3) their spectra in methanol. These latter are shown in Fig. 3 ; the phenolic acid isolated $\left(R_{F} 0.35\right.$ in propan-1-ol-aq. ammonia) gave a spectrum identical with that obtained with trans-p-coumaric acid, and the phenolic acid $\left(R_{F} 0.68\right)$ a spectrum identical with that of $c i s$ - $p$-coumaric acid. Finally, 


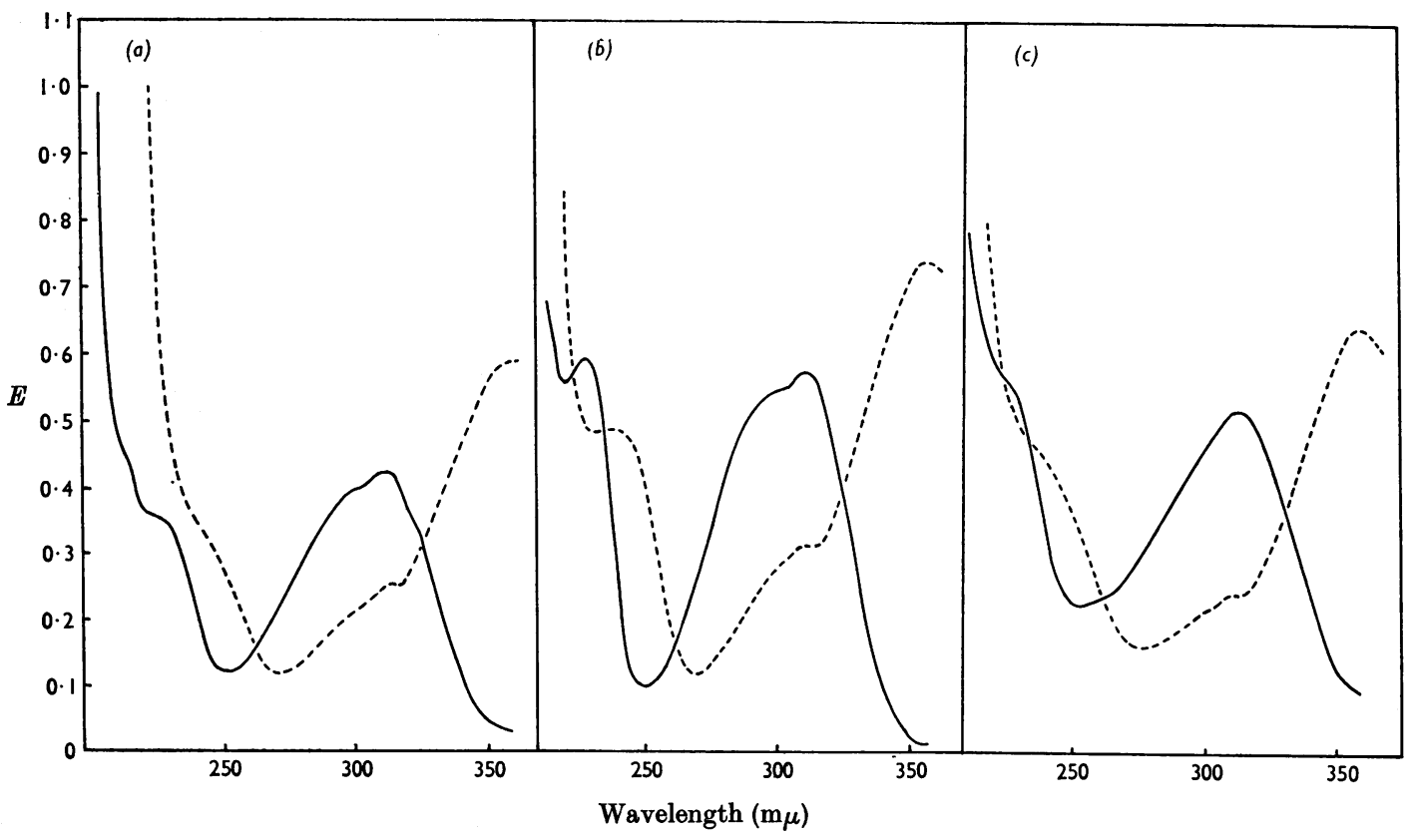

Fig. 2. Comparison of ultraviolet spectra of esters extracted from florets, with the methyl and glucose esters of p-coumaric acid in methanol. _- In neutral solution; --_-, after addition of KOH. (a) Ester from florets; (b) methyl ester; (c) glucose ester.

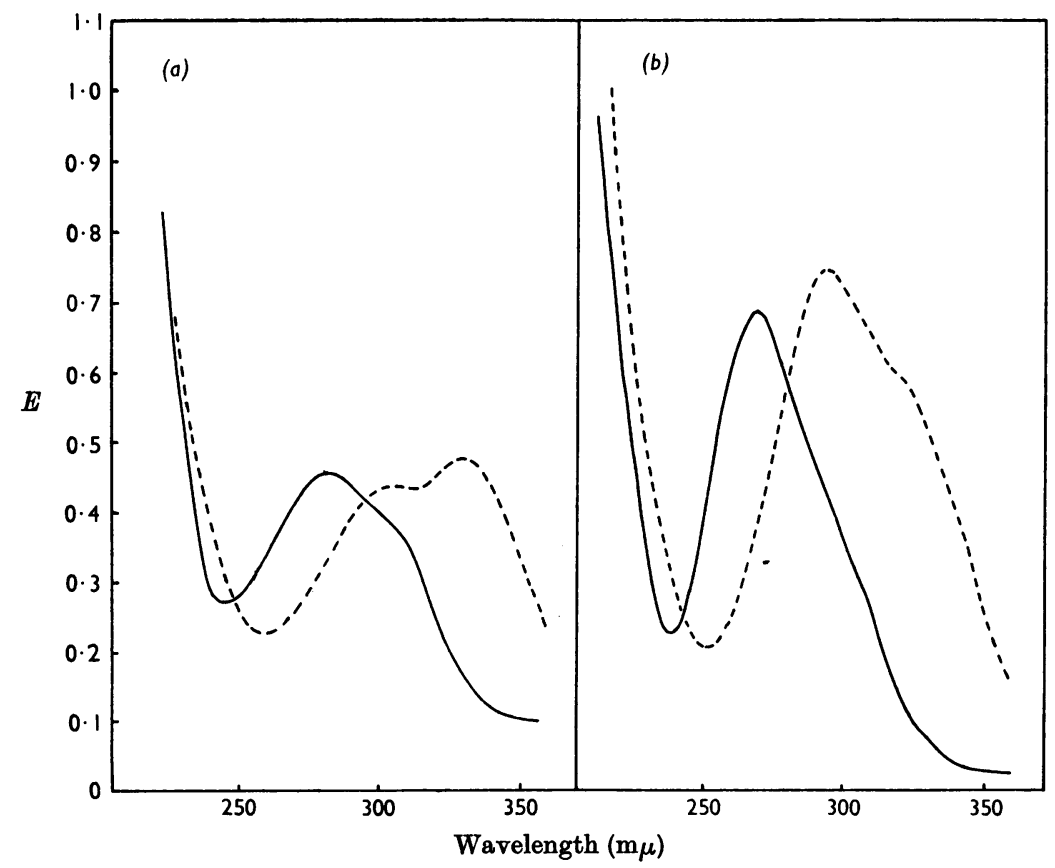

Fig. 3. Ultraviolet spectra in methanol of the phenolic components of the esters isolated from floret tissue after hydrolysis in $2 \mathrm{~N}-\mathrm{NaOH}$. —_, In neutral solution; ----, after addition of $\mathrm{KOH}$. (a) Phenolic component with $R_{F} 0.35$ in propan-1-ol-aq. $\mathrm{NH}_{3}$; (b) phenolic component with $R_{F} 0 \cdot 68$ in propan-1-ol-aq. $\mathrm{NH}_{3}$. 
Table 1. Relation between structure and activity of different phenols

Activity (\% of rate of formation of ethylene

Phenol

$p$-Hydroxybenzoic acid

$o$-Hydroxybenzoic acid

$m$-Hydroxybenzoic acid

$p$-Methoxybenzoic acid

$p$-Aminobenzoic acid

Benzoic acid

3,4-Dihydroxybenzoic acid

Cinnamic acid

$o$-Coumaric acid

$m$-Coumaric acid

$p$-Coumaric acid

$m$-Coumaric acid methyl ester

$p$-Coumaric acid methyl ester

$p$-Methoxycoumaric acid

Coumarin

Phenylacetic acid

$p$-Hydroxyphenylacetic acid

Phenylpropionic acid

p-Hydroxyphenylpropionic acid

$p$-Hydroxyphenylpyruvic acid

$p$-Hydroxyphenylglycollic acid

$p$-Hydroxyphenyl-lactic acid

Phenylalanine

p-Hydroxyphenylalanine

3,4-Dihydroxyphenylpropionic acid

3,4-Dihydroxycinnamic acid

4-Hydroxy-3-methoxycoumaric acid

2-Hydroxyacetophenone

4-Hydroxyacetophenone

2,4-Dihydroxyacetophenone

2,4,6-Trihydroxyacetophenone

2-Hydroxy-3-methoxyacetophenone

the silyl esters of $p$-coumaric acid and of the phenolic acid of the hydrolysed esters behaved identically after gas-liquid chromatography (temperature of emergence $190^{\circ}$ on analytical column programmed at $4^{\circ} / \mathrm{min}$. from $70^{\circ}$ ). Our tentative conclusion is that these components are either different or isomeric esters of $p$-coumaric acid. The nature of the other components of these $p$-coumaric acid esters has not as yet been definitely established, but it seems likely that they may be $p$-coumaryl esters containing either malic acid or tartaric acid, since these acids have been identified, as their silyl derivatives, in the hydrolytic products after hydrolysis with sodium hydroxide. The nature of the esters from the floret tissue proved to be mainly of academic interest, since all esters of $p$-coumaric acid so far tested (glucose, quinic and methyl) were found to be capable of replacing the natural esters as cofactors in the enzymic conversion of methional into ethylene. All were also found to be about three times as active in this respect as $p$-coumaric acid itself. We conclude that the $p$-coumaric acid moiety of the esters is the important constituent, and that the nature of the other component appears to be unimportant as far as the enzymic reaction is concerned.

Structure in relation to activity. The identification of the active component of fraction $C$, as an ester of $p$-coumaric acid, led us to examine the activity of a number of related phenols. Table 1 summarizes the results of a series of tests on the rate of production of ethylene from methional induced by the addition of various phenols or phenolic acids, in a concentration of $0.5 \mathrm{~mm}$, to an enzymic system containing the active component of fraction $A$. In these tests the rate of reaction induced by the addition of $p$-hydroxybenzoic acid was taken as being equal to 100 , this substance being selected as standard because it was one of the most active tested.

From the results listed it is clear that those phenols having a carbonyl group adjacent to a conjugated system are the most active. The tests also indicate that phenols having a carbonyl group in the side chain are active if this is adjacent to a conjugated system. Where a methylene group is interposed between these two groups the compound is inactive, as exemplified by the inactivity of $p$-hydroxyphenylacetic acid or $p$-hydroxyphenylpropionic acid compared with the activity of $p$ hydroxybenzoic acid or $p$-coumaric acid. Esterification of the carboxyl group does not diminish this activity, in fact it often enhances it; thus the methyl ester of $p$-coumaric acid promoted the reaction even more vigorously than $p$-coumaric acid.

The hydroxyl group in the benzene nucleus also appears to be essential, as indicated by the inactivity of benzoic acid, cinnamic acid or $p$ methoxycinnamic acid compared with the activity of $p$-hydroxybenzoic acid or $p$-coumaric acid. The amino group cannot be substituted for the hydroxyl group, since $p$-aminobenzoic acid is inactive. The greatest activity is usually found with the hydroxyl group in the para-position, the activity decreasing progressively para $>$ meta $>$ ortho. Thus the high activity of $p$-hydroxybenzoic acid contrasts with the lower activity of $m$-hydroxybenzoic acid and the absence of activity of salicylic acid. However, an exception to this is found with the hydroxyacetophenones, the $o$-hydroxy acid in this case being as active as the $p$-hydroxy acid.

The introduction of a second hydroxyl group into the benzene ring renders the compound completely inactive, and this applied to the phenones as well as the phenolic acid (see Table 1). In many cases the introduction of a second hydroxyl group had a definite inhibitory effect on the reaction (cf. Mapson \& Wardale, 1968). Thus caffeic acid, protocatechuic 
acid and dihydrocaffeic acid were all strongly inhibitory. Introduction of a second hydroxyl group into the benzene nucleus of the phenones does not, however, render the compound inhibitory as with the phenolic acids, though it does eliminate the catalytic activity.

$p$-Coumaric acid itself was inhibitory if used in higher concentrations. Fig. 4 shows that as the concentration of $p$-coumaric acid in the reaction mixture was increased from zero to $0.20 \mathrm{mg} . / \mathrm{ml}$. there was a progressive fall in the initial rate of the reaction. This we interpret as being due to the formation of caffeic acid from $p$-coumaric acid in

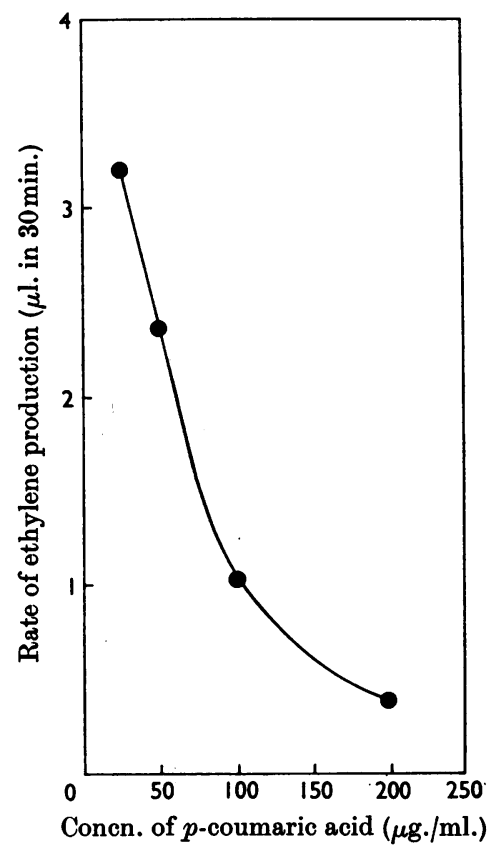

Fig. 4. Inhibitory effect of $p$-coumaric acid in higher concentrations. The reaction mixture was as described in Fig. 1, the acidic component of cofactor was supplied by fraction $A$ and $p$-coumaric acid was added as indicated. the presence of hydrogen peroxide and enzyme extract.

Component in fraction $A$. The component in fraction $A$ has not as yet been identified. It has, however, been isolated as a silyl ester, after preliminary purification by chromatography on paper with propan-1-ol-aq. ammonia (sp.gr. 0.88) (7:3, $\mathrm{v} / \mathrm{v})$ as solvent $\left(R_{F}, 0 \cdot 50\right)$. Elution with water was followed by the preparation of a silyl derivative, and this was isolated by gas-liquid chromatography on a preparative column of methyl silicone gum (SE 30) incorporating a flow splitter and programmed from $100^{\circ}$ at a rate of $4^{\circ} / \mathrm{min}$. Under these conditions the temperature of emergence of the silyl derivative was $135-137^{\circ}$.

Yang (1967) has described a model system, comprising horseradish peroxidase, hydrogen peroxide, a phenolic compound and sulphite, that readily produces ethylene from methional. The resemblance of this system to the one described here prompted us to determine whether sulphite could replace the acidic component of fraction $A$ in the enzymic system isolated from the florets. The results (Table 2) show that sulphite is effective in replacing this acid in producing ethylene from methional, and further that the acid itself can replace the sulphite in the model system described by Yang (1967). Our unknown acid is not, however, sulphite; it is organic and non-volatile as the free acid, and gives a negative test for sulphite in the nitroprusside-ferrocyanide reaction described by Feigl (1947). The unknown acid does, however, contain sulphur, but the compound is not identical with any of the acids commonly found in plant tissues. In the floret tissue it is present in a concentration of about $\mathbf{0 . 7 \%}$ of the total organic acids extractable with diethyl ether.

\section{DISCUSSION}

Yang (1967) suggested a reaction mechanism for the production of ethylene from methional by horseradish peroxidase, phenols and sulphite in the

Table 2. Replacement of unknown acid by sulphite as cofactor

All systems contained $8 \mathrm{ml}$. of $\mathrm{KH}_{2} \mathrm{PO}_{4}-\mathrm{Na}_{2} \mathrm{HPO}_{4}$ buffer, pH 6.5 (0.1 M), EDTA (2 mM), glucose (1\%, w/v), fungal glucose oxidase (10 $\mu \mathrm{g}$.), methional (1 mM), $p$-hydroxybenzoic acid (1 mg.) and either the methional-cleaving enzyme from the florets or horseradish peroxidase $(40 \mu \mathrm{g}$.) in a total volume of $10 \mathrm{ml}$.

Ethylene formed ( $\mu$ l.)

Enzyme added

Methional-cleaving enzyme from florets

Horseradish peroxidase

\section{Cofactor added Time (hr.) $\quad$...}

$\mathrm{NaHSO}_{3}(0 \cdot 1 \mathrm{~mm} ; 2 \mu$ equiv.)

$\mathrm{NaHSO}_{3}(0.5 \mathrm{~mm} ; 10 \mu$ equiv.)

$\mathrm{NaHSO}_{3}$ (2.0 mM; $40 \mu$ equiv.)

Control (unknown acid) (1.0 $\mu$ equiv.*)

Unknown acid (1.0 $\mu$ equiv.*)

\begin{tabular}{lll}
\hline 0.25 & 0.5 & 1.0 \\
1.18 & 1.8 & 1.8 \\
4.55 & 7.4 & 8.8 \\
0.29 & 0.81 & 4.3 \\
4 & 6.5 & 6.5 \\
4.4 & 6.4 & 7
\end{tabular}

\footnotetext{
* On the assumption that the unknown is a monobasic acid.
} 
presence of hydrogen peroxide, involving the production of free radicals, which accept electrons from the sulphur atom of methional with the subsequent decomposition of methional into dimethyl disulphide, ethylene and formic acid. Until the nature of the acidic component of the cofactor for the enzyme system described here has been established, it seems worthless to speculate further as to the chemical mechanism underlying these reactions. However, it is worth noting the results of Tomaszewski \& Thimann (1966) on the interactions of phenolic acids on auxin-induced growth in Avena coleoptiles and pea epicotyls. The stimulation or depression of growth observed they related to the ability of individual phenolic acids to stimulate or inhibit the enzymic decarboxylation of indol-3-ylacetic acid. Our results on the effect of the phenolic acids on the enzymic synthesis of ethylene from methional closely resemble those of Tomaszewski \& Thimann (1966). Thus the polyphenols, e.g. chlorogenic acid, caffeic acid and sinapic acid, synergize indol-3-ylacetic acid-induced growth by counteracting decarboxylation of indol3-ylacetic acid, whereas the monophenols, e.g. $p$-coumaric acid and $p$-hydroxybenzoic acid, stimulate decarboxylation of indol-3-ylacetic acid under those conditions where they depress growth. The polyphenols chlorogenic acid and sinapic acid, as well as the dihydroxyphenols catechol or resorcinol, strongly inhibit the synthesis of ethylene from methional (Mapson \& Wardale, 1968). We have now demonstrated that certain $p$-hydroxyphenols, e.g. $p$-coumaric acid and derivatives and $p$-hydroxybenzoic acid, are necessary components of the enzymic system catalysing the conversion of methional into ethylene.

As with the auxin experiments the introduction into a phenolic nucleus of a second hydroxyl group converts a cofactor into one that inhibits the enzymic reaction. In our experiments (Mapson \& Wardale, 1968) we tentatively suggested that the dihydroxyphenols, oxidized in the presence of peroxide, inhibit the formation of ethylene from methional by competing with the methionalcleaving enzyme for the peroxide generated by the plant oxidase. Whether this is the correct interpretation remains to be proved.

Note added in proof. The unknown acid has been identified as methanesulphinic acid by comparison with authentic methanesulphinic acid. The identity of the two acids has been established by mass spectrometry, gas-liquid chromatography of the trimethylsilyl derivatives, paper chromatography of the ammonium salts and identical response in the enzymic production of ethylene from methional.

\section{REFERENCES}

Dalgleish, C. E., Horning, E. C., Horning, M. G., Knox, K. L. \& Yarger, K. (1966). Biochem. J. 101, 792.

Feigl, F. (1947). Qualitative Analysis by Spot Tests, 3rd ed., p. 231.

Mapson, L. W. \& Wardale, D. (1967). Biochem.J. 102, 574.

Mapson, L. W. \& Wardale, D. (1968). Biochem. J. 107, 433.

Tomaszewski, M. \& Thimann, K. V. (1966). Plant Physiol. 41, 1443.

Yang, S. F. (1967). Arch. Biochem. Biophys. 122, 481. 